\title{
DEVELOPMENT OF A FEMININITY ESTIMATOR USING SPEAKER RECOGNITION TECHNIQUES FOR VOICE THERAPY OF GENDER IDENTITY DISORDER CLIENTS
}

\author{
Nobuaki MINEMATSU ${ }^{1}$, Kazutaka MARUYAMA ${ }^{1}$, Kyoko SAKURABA ${ }^{2}$ Keikichi HIROSE $^{1}$, \\ Niro TAYAMA ${ }^{3}$, Satoshi IMAIZUMI ${ }^{4}$, Toshio YAMAUCHI ${ }^{5}$ \\ ${ }^{1}$ The University of Tokyo, ${ }^{2}$ Kiyose-shi Welfare Center for the Handicapped, \\ ${ }^{3}$ International Medical Center of Japan, ${ }^{4}$ Prefectural University of Hiroshima, \\ ${ }^{5}$ Saitama Medical University \\ \{mine, maruyama, hirose\}@gavo.t.u-tokyo.ac.jp, sakuraba@mtd.biglobe.ne.jp
}

\begin{abstract}
This paper describes the development of an estimator of perceptual femininity (PF) of an input utterance using speaker recognition techniques. The estimator was designed for its clinical use and the target speakers are Gender Identity Disorder (GID) clients, especially MtF (Male to Female) transsexuals. The voice therapy for MtFs is composed of three kinds of training; 1) raising the baseline $\mathrm{F}_{0}$ range, 2) changing the baseline voice quality, and 3 ) enhancing $\mathrm{F}_{0}$ dynamics to produce an exaggerated intonation pattern. The first two focus on static acoustic properties of speech and the voice quality is mainly controlled by size and shape of the articulators, which can be acoustically characterized by the spectral envelope. Gaussian Mixture Models (GMM) of $\mathrm{F}_{0}$ values and spectrums were built separately for biologically male speakers and female ones. Using the four models, PF was estimated automatically for each of 142 utterances of 111 MtFs. The estimated values were compared with the PF values obtained through listening tests. Results showed very high correlation ( $R=0.86$ ), which is comparable to the intra-rater correlation.
\end{abstract}

Index Terms - Femininity, GID, speaker recognition, GMM

\section{INTRODUCTION}

Advanced speech technologies are applied not only for man-machine interface and entertainment but also for medical treatment and language education. Many works were done for developing cochlea implants and artificial larynxes and, recently, the technologies have been applied to realize an on-line screening test of laryngeal cancer [1] as well as an on-line test of pronunciation proficiency of foreign languages [2]. The present paper examines the use of the technologies for another medical treatment; voice therapy for GID clients.

A GID individual is one who strongly believes that his or her true psychological gender identity is not his or her biological or physical gender, i.e., sex. In most of the cases, GID individuals live for years trying to conform to the social role required by their biological gender, but eventually seek medical and surgical help as well as other forms of therapy in order to achieve the physical characteristics and the social role of the gender which they feel to be their true one. In both cases of FtMs (Female-to-Male) and MtFs, many of them take hormone treatment to make physical change of their bodies and the treatment is certainly effective for both cases. However, it is known that the hormone treatment brings about sufficient change of the voice quality only for FtMs. Considering that the voice quality is controlled by physical conditions of the articulators, the vocal folds and the vocal tract are presumed to retain their pretreatment size and shape in the case of MtFs. To overcome this hardship and mainly to shift up the baseline $\mathrm{F}_{0}$ range, some $\mathrm{MtFs}$ take surgical treatment. Although the $\mathrm{F}_{0}$ range is certainly raised in the new voice, as far as the third author knows, it is a pity that the naturalness is decreased in the new voice instead. Further, many clinical papers and engineering papers on speech synthesis claim that raising the $\mathrm{F}_{0}$ range alone does not produce good femininity $[3,4,5]$. Since shape of the vocal tract has a strong effect on the voice quality, good control of the articulators has to be learned to achieve good femininity. Considering small effects of the hormone treatment and the surgical treatment on $\mathrm{MtF}$ clients, we can say that the most effective and least risky method to obtain good femininity is taking voice training from speech therapists or pathologists with good knowledge of GID.

\section{WHY FEMININITY ESTIMATOR?}

In the typical therapy, the following three methods are used. 1) raising the baseline $\mathrm{F}_{0}$ range, 2) changing the baseline voice quality, and 3) enhancing $F_{0}$ dynamics to produce an exaggerated intonation pattern. One of the most difficult things in the voice therapy lies not on a client's side but on a therapist's side, i.e., accurate and objective evaluation of the client's voice. It is often said that as synthetic speech samples are presented repeatedly, even expert speech engineers tend to perceive better naturalness in the samples, known as habituation effect. This is the case with good therapists. To avoid this effect and evaluate the femininity unbiasedly, listening tests with novice listeners are desirable. But the tests take a long time and a large cost. Further, in most of the cases, GID clients are eager to know how they sound to novice listeners. Some clients, not so many, claim that they sound feminine enough although they sound less feminine to anybody else. The objective evaluation of their voices is very effective to let these clients know the truth. Then, in this study, a listening test simulator was developed by automatically estimating the femininity which novice listeners would perceive if they heard the samples.

Among the above three methods, the first two ones focus on static acoustic properties and the last one deals with dynamic $\mathrm{F}_{0}$ control. The dynamic control of $F_{0}$ for various speaking styles is a very challenging task in speech synthesis research and, therefore, we only focused on the femininity created by the $\mathrm{F}_{0}$ range and the voice quality. GMM modeling of $F_{0}$ values and that of spectrums were done separately for biologically male speakers and female ones. By using the four models, the estimator was developed. In this paper, as well as the experimental results of the femininity estimation, some merits and demerits of using the estimator in voice therapy are described. 


\section{GMM-BASED MODELING OF FEMININITY}

\subsection{Modeling femininity with isolated vowels}

Questions of acoustic cues of good femininity were often raised in previous studies $[6,7,8,9]$. Acoustic and perceptual analysis of speech samples of biologically male and female speakers and those of $\mathrm{MtF}$ ones were done and the findings lead to the three kinds of methods in the previous section. About the voice quality, as far as the authors know, all the studies focused on isolated vowel utterances and formant frequencies were extracted to estimate the femininity. It is true that, even from a single /a/ utterance, it is possible to estimate vocal tract length [10] and then, the femininity. It is also true, however, that even if a client can produce very feminine isolated vowels with careful articulation, it does not necessarily mean that the client can produce continuous speech with good femininity. This is the case with foreign language pronunciation. Even if a learner can produce very good isolated vowels, the learner is not always a good speaker of the target language in normal speech communication. This is partly because good control of prosody is required in continuous speech. However, we consider another reason that so much attention cannot be paid to every step of producing vowels in a sentence. We can say that the desired tool for MtF voice therapy is an estimator of the femininity from continuous speech. Here, we have a fundamental problem. With the analysis methods used in the previous studies, it is difficult to estimate the femininity from continuous speech because formant frequencies change not only due to the femininity but also due to phonemic contexts of the vowel.

\subsection{Modeling femininity with continuous speech}

This problem can be solved by using GMM-based speaker recognition techniques. In continuous speech, various phonemes are found and the phonemes naturally cause spectral changes. If the utterance has sufficient spectral variations, averaging the spectrum slices over time can effectively cancel the spectral changes caused by the phonemic variation. The resulting average pattern of spectrum comes to have a statically biased form of spectrum, which is considered to characterize the speaker identity and the stationary channel. In GMM-based speaker recognition, the average pattern is modeled not as a single spectrum slice but as a mixture of Gaussian distributions, where the spectrum is often represented as cepstrum vector.

With speech samples of any text spoken by a large number of female speakers, a GMM was trained to characterize the spectrumbased femininity, $M_{F}^{s}$. With male speakers, a GMM for the masculinity was trained, $M_{M}^{s}$. Using both models, the eventual spectrum-based femininity for a given cepstrum vector $o, F^{s}(o)$, was defined as the following formula $[11,12]$;

$$
F^{s}(o)=\log \mathcal{L}\left(o \mid M_{F}^{s}\right)-\log \mathcal{L}\left(o \mid M_{M}^{s}\right) .
$$

Similar models are trained for the $\mathrm{F}_{0}$-based femininity and masculinity, $M_{F}^{f}$ and $M_{M}^{f}$;

$$
F^{f}(o)=\log \mathcal{L}\left(o \mid M_{F}^{f}\right)-\log \mathcal{L}\left(o \mid M_{M}^{f}\right) .
$$

Integration of the four models, $M_{F}^{s}, M_{M}^{s}, M_{F}^{f}$, and $M_{M}^{f}$ can be done through generalizing the above formulae;

$$
\begin{aligned}
F(o)= & \alpha \log \mathcal{L}\left(o \mid M_{F}^{s}\right)+\beta \log \mathcal{L}\left(o \mid M_{M}^{s}\right)+ \\
& \gamma \log \mathcal{L}\left(o \mid M_{F}^{f}\right)+\varepsilon \log \mathcal{L}\left(o \mid M_{M}^{f}\right)+C,
\end{aligned}
$$

where $\alpha, \beta, \gamma, \varepsilon$, and $C$ are calculated so that the $F(o)$ can predict perceptual femininity (PF) of $o$ the best. The PF scores were obtained in advance through listening tests with novice listeners.

\section{FEMININITY LABELING OF MTF SPEECH CORPUS}

\subsection{MtF speech corpus}

A speech corpus of $111 \mathrm{Japanese} \mathrm{MtF}$ speakers was built, some of whom sounded very feminine and others sounded less feminine and needed additional therapy. Each speaker read the beginning two sentences of "Jack and the beanstalk" with natural speaking rate and produced isolated Japanese vowels of /a, i, u, e, o/. The two sentences had 39 words. All the speech samples were recorded and digitized with 16 bit and $16 \mathrm{kHz}$ AD conversion. Some clients joined the recording twice; before and after the voice therapy. Then, the total number of recordings was 142 . For reference, 17 biologically female Japanese read the same sentences and produced the vowels.

\subsection{Perceptual femininity labeling of the corpus}

All the sentence utterances were randomly presented to 6 male and 3 female adult Japanese subjects through headphones. All the subjects were in their 20 s with normal hearing and they were very unfamiliar with GID. The subjects were asked to judge subjectively how feminine each utterance sounded and write down a score using a 7-degree scale, where +3 corresponded to the most feminine and -3 did to the most masculine. Some speech samples of biological female speakers were used as dummy samples. Every subject joined the test twice and 18 femininity judgments by 9 subjects were obtained for each utterance. Figure 1 shows histogram of the averaged $\mathrm{PF}$ scores for the individual $\mathrm{MtF}$ utterances. Although some utterances still sounded rather masculine, a good variance of PF was found in the corpus. The averaged PF of biological female speakers was 2.74. While, in Figure 1, the averaging operation was done over all the subjects, in the following section, it will also be done dependently on the subject's biological gender.

\subsection{Intra-subject and inter-subject judgment agreement}

Agreement between the judgments within a subject was examined. Each subject joined the test twice and the correlation between the two sessions was calculated for each. The averaged correlation over the subjects was 0.80 , ranging from 0.48 to 0.91 . If the subject with the lowest correlation is deleted, the averaged intra-subject correlation was recalculated as 0.84 ( 0.79 to 0.91$)$.

$P F$ scores by a subject were defined as the scores averaged over the two sessions. Using these scores, the judgement agreement between two subjects was analyzed. The agreement between a female and another female was averaged to be 0.76 , ranging from 0.71 to 0.83 . In the case of the male subjects, the agreement was averaged to be 0.75 , ranging from 0.59 to 0.89 . The agreement between a female and a male was 0.71 on average, ranging from 0.60 to 0.79 .

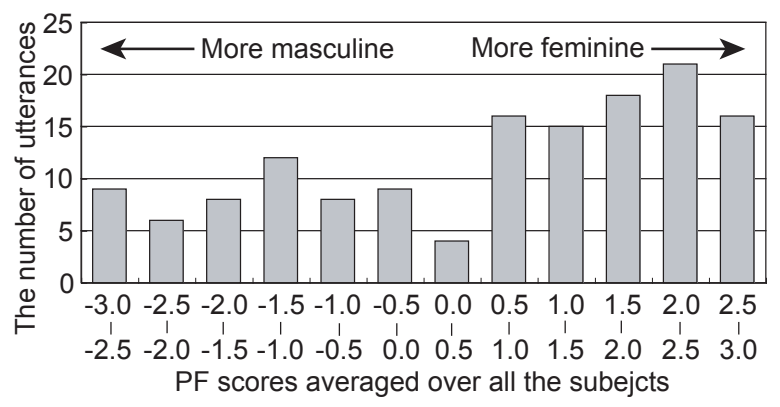

Fig. 1. Histogram of the averaged PF scores of the 142 utterances 
Table 1. Acoustic conditions of the analysis

\begin{tabular}{ll}
\hline sampling & $16 \mathrm{bit} / 16 \mathrm{kHz}$ \\
window & $25 \mathrm{~ms}$ length and $10 \mathrm{~ms}$ shift \\
parameters & $\mathrm{MFCC}$ with its $\Delta$ and $\Delta \Delta$ for $M_{F}^{s}$ and $M_{M}^{s}$ \\
& $\log \mathrm{F}_{0}$ for $M_{F}^{f}$ and $M_{M}^{f}$ \\
$\mathrm{GMM}$ & mixture of 16 Gaussian distributions
\end{tabular}

Table 2. Correlation of $F^{s}$ and $F^{f}$ with the three PF scores

\begin{tabular}{cccc}
\hline & female PF & male PF & averaged PF \\
\hline$F^{s}$ & 0.71 & 0.70 & 0.73 \\
$F^{f}$ & 0.67 & 0.76 & 0.74 \\
\hline
\end{tabular}

Table 3. Correlation of $F$ with the three PF scores

\begin{tabular}{cccc}
\hline & female PF & male PF & averaged PF \\
\hline$F$ & 0.78 & 0.86 & 0.84 \\
\hline
\end{tabular}

Some strategic differences in the judgment may be found between the two sexes, which will be discussed later.

$P F$ scores by the female were defined as the averaged scores over the three female subjects. Similarly, $P F$ scores by the male were defined. The correlation between the two sexes was 0.87 , which is very high compared to the averaged inter-subject correlation between the two sexes (0.71). This is because of the double averaging operations, which could reduce inevitable variations in the judgments effectively.

Now, we have 12 different kinds of PF scores; 9 from the 9 subjects, 2 as the scores by the male and the female, and the other one obtained by averaging the male score and the female one. In the following sections, the correlations between the original PF scores and the estimated PF scores, defined in Section 3.2, are investigated.

\section{TRAINING OF $M_{F}^{S}, M_{M}^{S}, M_{F}^{F}$ AND $M_{M}^{F}$}

As described in Section 3.2, automatic estimation of the femininity is examined based on GMM-based modeling. As speech samples for training, JNAS (Japanese Newspaper Article Sentences) [13] speech database, 114 biological males and 114 biological females, was used. The number of sentences was 3,420 for each sex. Table 1 shows acoustic conditions used in the analysis. For the spectrumbased GMMs of $M_{F}^{s}$ and $M_{M}^{s}$, silence removal was carried out from the speech files and 12 dimensional MFCCs with their $\Delta$ and $\Delta \Delta$ of only the remaining speech segments were used. For the $\mathrm{F}_{0}$-based GMMs of $M_{F}^{f}$ and $M_{M}^{f}, \log \mathrm{F}_{0}$ values were utilized.

\section{AUTOMATIC ESTIMATION OF FEMININITY}

\subsection{Simple estimation based on $F^{s}$ and $F^{f}$}

For each of the $142 \mathrm{MtF}$ utterances, their femininity scores were estimated using $F^{s}$ and $F^{f}$. The estimated scores were compared with the 12 different PF scores and the correlation was calculated separately. The averaged correlation over the first $9 \mathrm{PF}$ scores is 0.64 for $F^{s}$ and 0.66 for $F^{f}$. Table 2 shows the correlation of $F^{s}$ the $F^{f}$ with the other three PF scores; the male and the female scores and their averaged one. While the female $\mathrm{PF}$ is more highly correlated with $F^{s}$ than $F^{f}$, the male PF is more highly correlated with $F^{f}$ than $F^{s}$. This may imply different strategies of judging the femininity between the male and the female subjects. It seems that the male tend to perceive the femininity better in high pitch of the voice. This finding is accordant with the results obtained in a previous study
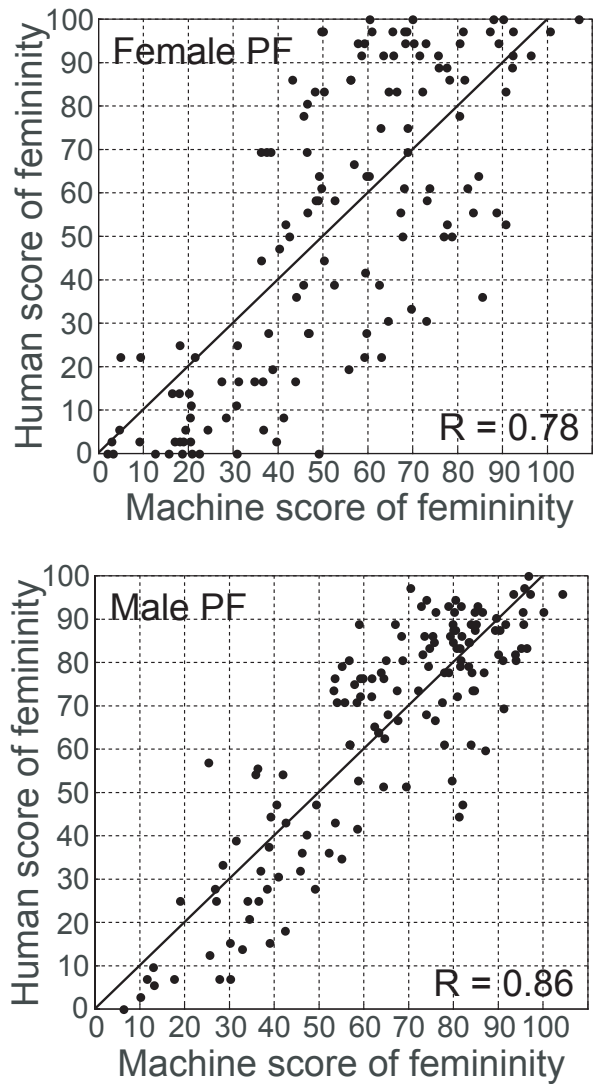

Fig. 2. Correlation between the original and the estimated scores

done by the second author [14]. In the study, it was shown that male listeners tended to assign higher femininity scores to speech samples with higher pitch.

\subsection{Integrated estimation with weighting factors}

Linear regression analysis was done to predict the $12 \mathrm{PF}$ scores using the four models, where the PF scores were converted to have a range from 0 (the most masculine) to 100 (the most feminine). As shown in Equation 3, four weighting factors and one constant term were calculated to minimize the prediction error.

The averaged multiple correlation over the first 9 PF scores was increased up to 0.76 . Table 3 shows the multiple correlation coefficients with the male PF score, the female one, and the averaged one. Figure 2 graphically shows the correlation with the first two PF scores. Here, for utterance(s) of an MtF speaker, the weighting factors were calculated using utterances of the other MtF speakers and then, the femininity score(s) of utterance(s) of that MtF speaker were estimated. Namely, the estimation was done in a speaker-open mode. Considering magnitude of the intra- and inter-subject correlations of $\mathrm{PF}$, we can say that $F(o)$ is a very good estimator of PF.

\subsection{Discussions}

The four weighting factors and the constant term in Equation 3 show different values for the $12 \mathrm{PF}$ scores. The difference in values between two subjects characterizes the difference in judging strategies between them. Table 4 shows 12 patterns of $\alpha, \beta, \gamma, \varepsilon$, and $C$ with the multiple correlation coefficient $(R)$. As was found in Section 6.1, 
Table 4. Values of the weighting factors and the constant term

\begin{tabular}{lcccccc}
\hline & $\alpha$ & $\beta$ & $\gamma$ & $\varepsilon$ & $C$ & $R$ \\
& $M_{F}^{s}$ & $M_{M}^{s}$ & $M_{F}^{f}$ & $M_{M}^{f}$ & & \\
\hline F1 & 0.154 & -0.143 & 0.068 & -0.017 & 1.301 & 0.77 \\
F2 & 0.133 & -0.126 & 0.086 & -0.012 & 1.005 & 0.68 \\
F3 & 0.087 & -0.112 & 0.167 & -0.011 & -0.742 & 0.72 \\
M1 & 0.074 & -0.069 & 0.120 & -0.009 & 1.091 & 0.82 \\
M2 & 0.035 & -0.024 & 0.176 & -0.030 & 1.479 & 0.73 \\
M3 & 0.076 & -0.085 & 0.150 & 0.028 & 0.446 & 0.80 \\
M4 & 0.034 & -0.035 & 0.172 & -0.005 & 0.941 & 0.76 \\
M5 & 0.090 & -0.084 & 0.141 & 0.023 & 1.237 & 0.86 \\
M6 & 0.100 & -0.090 & 0.107 & 0.047 & 1.389 & 0.70 \\
female PF & 0.125 & -0.127 & 0.107 & -0.013 & 0.521 & 0.78 \\
male PF & 0.068 & -0.064 & 0.144 & 0.009 & 1.097 & 0.86 \\
average PF & 0.097 & -0.096 & 0.126 & -0.002 & 0.809 & 0.84 \\
\hline
\end{tabular}

clear difference was found between the female and the male subjects. The female tend to focus on spectral properties $(\alpha=0.125$ and 0.068 for female PF and male PF), while the male tend to focus on pitch ( $\gamma=0.107$ and 0.144 for female PF and male PF). In this sense, F3's judgment is very male because she emphasized pitch $(\gamma=0.167)$ and de-emphasized spectral properties $(\alpha=0.085)$. In Table 3 and Figure 2, the multiple correlation was not so high for the female PF scores $(R=0.78)$ and this can be considered probably because of F3.

For every PF score, the absolute value of $\alpha$ and that of $\beta$ are similar. $\alpha$ and $\beta$ of the female PF are 0.125 and -0.127 . Those of the male PF are 0.068 and -0.064 . This directly means that, with spectral properties of the voice, it is as important to shift the client's voice closer to the female region as to shift the voice away from the male region. On the contrary, the absolute value of $\gamma$ and that of $\varepsilon$ show a large difference. $\gamma$ and $\varepsilon$ of the female PF are 0.107 and -0.013 . Those of the male PF are 0.144 and 0.009 . In every case, $\varepsilon$ takes a very small value, near to zero, compared to $\gamma$. This indicates that, as for $\mathrm{F}_{0}$, although it is important to shift the voice into the female region, it matters very little if the voice is still located in the male region. This asymmetric effects of spectrum and $\mathrm{F}_{0}$ can be summarized as follows by using ideas of bonus and penalty. If the voice is closer to the female region, larger bonus is given and if the voice is closer to the male region, larger penalty is given. Although both bonus and penalty should be considered with spectral properties of the voice, only bonus is good enough with its $\mathrm{F}_{0}$ properties.

\section{ACTUAL USE OF THE ESTIMATOR IN VOICE THERAPY - MERITS AND DEMERITS -}

The second author has used the estimator in her voice therapy for $\mathrm{MtF}$ clients since Feb. 2006. It was found that, when biologically male speakers without any special training pretended to be female, it was very difficult to get a score higher than 80 . However, it is very interesting that good $\mathrm{MtF}$ speakers, who can change their speaking mode voluntarily from male to female, could have the estimator show a very low score (very masculine) and a very high score (very feminine) at their will. Since the estimator is focusing on only static acoustic properties, we consider that these MtFs have two baseline shapes of the vocal tract, which may be realized by different positioning of the tongue, and two baseline ranges of $\mathrm{F}_{0}$. In this sense, the estimator helped new clients a great deal seek for another baseline of the vocal tract shape and/or that of $F_{0}$ range through many try-and-errors. Needless to say, quantitative and objective evaluation of their trials motivated the clients very well.

Only the focus on static acoustic properties naturally caused some problems. As described in Section 1, by producing a rather exaggerated pattern of intonation, listeners tend to perceive higher femininity. Although this exaggeration is a good technique to obtain high PF in the voice, the estimator completely ignores this aspect and then, some clients showed unexpectedly high scores or low scores. In actual therapies, what is possible and what is impossible with the estimator should be correctly instructed to the clients especially when they evaluate their voices by themselves.

\section{CONCLUSIONS}

This paper described the development of an automatic estimator of the perceptual femininity from continuous speech with speaker recognition techniques. Spectrum-based and Fo-based GMMs were separately trained with biological male and female speakers. By integrating these models, the estimator was built. The correlation of the estimated values with the perceptual femininity scores originally obtained through listening tests was 0.86 , comparable to the intrarater correlation. Some analyses were done about sexual differences of the femininity judgment and some strategic differences in the use of spectrum-based cues and F0-based cues were shown. The male subjects tended to give higher scores to the voices with higher pitch. Further, it was indicated independently of the subject's sex that the penalty of the $\mathrm{F}_{0}$ range being still in the male region is remarkably small. As future work, we are planning to take MRI pictures of a good MtF speaker's control of the articulators when producing feminine vowels and masculine ones. We hope that the estimator will help many MtF clients improve the quality of their lives.

\section{REFERENCES}

[1] H. Mori et al., "Internet-based acoustic voice evaluation system for screening of laryngeal cancer," J. Acoustic Society of Japan, vol.62, no.3, pp.193-198 (2006, in Japanese)

[2] J. D. Jong et al., "Relating phonepass scores overall scores to the council of europe framework level descriptors," Proc. EUROSPEECH, pp.2803-2806 (2001)

[3] R. C. Bralley et al., "Evaluation of vocal pitch in male transsexuals," J. Communication Disorder, vol.11, pp.443-449(1978)

[4] L. E. Spencer, "Speech characteristics of MtF transsexuals: a perceptual and acoustic study," Folia phoniat., vol.40, pp.31-42 (1988)

[5] K. H. Mount et al., "Changing the vocal characteristics of a postoperative transsexual patient: a longitudinal study," J. Communication Disorder, vol.21, pp.229-238 (1988)

[6] S. Bennett, "Acoustic correlates of perceived sexual identity in preadolescent children's voices,” J. Acoust. Soc. Am., vol.66, no.4, pp.9891000 (1979)

[7] M. L. Andrews et al., "Gender presentation: perceptual and acoustical analyses of voice," J. Voice, vol.11, no.3, pp.307-313 (1997)

[8] M. P. Gelfer, "Comparison of acoustic and perceptual measures of voice in $\mathrm{MtF}$ transsexuals perceived as female vs. those perceived as male," J. Voice, vol.14, no.1, pp.22-33 (2000)

[9] V. I. Wolfe, "Intonation and fundamental frequency in MtF TS," J. Speech Hearing Disorders, vol.55, pp.43-50 (1990)

[10] A. Paige et al., "Calculation of vocal tract length," IEEE Trans. on Audio and Electroacoustics , vol.AU-18, no.3, pp.268-270 (1970)

[11] A. E. Rosenberg et al., "Speaker background models for connected digit password speaker verification," Proc. ICASSP, pp.81-84 (1996)

[12] L. P. Heck et al., "Handset-dependent background models for robust text-independent speaker recognition," Proc. ICASSP, pp.1071-1074 (1997)

[13] http://www.mibel.cs.tsukuba.ac.jp/jnas/

[14] K. Sakuraba et al., "Sexual difference between male and female listeners in the perceptual test with the voice produced by MtF transsexuals,' Proc. Spring Meeting of ASJ, 2-P-4, pp.337-338 (2004, in Japanese) 